\title{
Discursos en torno a la administración del conflicto y su relación con la convivencia escolar
}

\section{Discussions regarding the administration of the conflict and its relationship with school coexistence}

DOI: http://dx.doi.org/10.17981/cultedusoc.8.2.2017.02

Paula Ascorra ${ }^{1}$, Paula Vásquez ${ }^{2}$, Nicolás Passalacqua ${ }^{3}$, Claudia Carrasco ${ }^{4}$, Verónica López ${ }^{5}$, Carmen Gloria Núñez y Maria Francisca Álvarez Figueroa ${ }^{7}$

\section{Resumen}

Se examina la relación entre formas de administrar conflicto y estilos de convivencia escolar (CE) en dos liceos públicos chilenos. Se hipotetiza que el tipo de administración implementado incidirá en la forma y características de la CE. Mediante microetnografías y análisis de contenido, distinguimos dos formas de administrar el conflicto: la tecnocrática positivista y la crítica; y tres estilos de CE: funcional autoritaria, funcional democrática y democrática sociocrítica. Los resultados muestran en ambos casos, una administración tecnocrática positivista, caracterizada por un alto control comportamental de estudiantes y profesores, alta normatividad con acentos en aspectos punitivos, y estrategias de eliminación e invisibilización de los problemas que se presentan.

Palabras Claves: Administración del conflicto, convivencia escolar, autoritarismo, democracia

\footnotetext{
${ }^{1}$ Pontificia Universidad Católica de Valparaíso. Centro de Investigación para la Educación Inclusiva.

${ }^{2}$ Pontificia Universidad Católica de Valparaíso.

${ }^{3}$ Pontificia Universidad Católica de Valparaíso.

${ }^{4}$ Universidad de Playa Ancha.

${ }^{5}$ Pontificia Universidad Católica de Valparaíso. Centro de Investigación para la Educación Inclusiva.

${ }^{6}$ Pontificia Universidad Católica de Valparaíso.

${ }^{7}$ Sicologa de la Pontificia Universidad Católica de Valparaíso. Master of Science in Human Resource Management - University of Liverpool. Centre of Research for Inclusive Education.Correo: eduinclusiva.cl y maria.alvarez@pucv.cl
} 


\begin{abstract}
We examine the relationship between conflict management and school climate styles in two Chilean public schools. It is hypothesized that the type of administration implemented will influence the form and characteristics of the school life. Through microethnographies, and content analysis, we distinguished two forms of conflict management: the positivist and critical technocratic; and three styles of school coexistence: functional authoritarian, functional democratic and sociocritical democracy. The results show, in both cases, a technocratic positivist administration, characterized by a high behavioral control of students and teachers, high normativity with accents in punitive aspects, and strategies of elimination and invisibilization of the problems that are presented.
\end{abstract}

Keywords: Conflict management, school coexistence, authoritarism, democracy

\title{
Introducción
}

La temática del conflicto en los procesos educativos, se ha vuelto una estrategia para mejorar el clima relacional y organizativo en los contextos escolares (Arón, \& Milicic, 1999; Fierro, 2013; Llaña, 2011; Tuvilla, 2007). Asimismo, la CE se ha convertido en el eje central de las políticas públicas en materia de educación tanto a nivel nacional como internacional. Esta categoría, ha sido abordada desde diversos enfoques, tales como la Educación para la Paz y los Derechos Humanos, Educación y Democracia, así también desde el campo de la Inclusión, el Género, la Educación Valórica, Intercultural y Cívica (Fierro, 2012; Ianni, 2003; Magendzo, Toledo, \& Gutierrez, 2012; Tuvilla, 2007).

Todas estas perspectivas se caracterizan por instaurarse tanto en la cotidianeidad de la escuela como en el marco de las políticas públicas propias de cada país. Chile no se encuentra exento de ellas, la Política Nacional de Convivencia Escolar (2011), muestra una tendencia hacia el área de Educación y Democracia, que se reflejada en la intención de democratizar las escuelas y liceos del país (Aceituno, Muñoz y Vásquez, 2012; Delannoy, 2003; González, 2006; Santos Guerra, 2004).

Así, el objetivo de este artículo es comprender la relación entre las formas en que dos establecimientos educacionales administran el conflicto y los estilos de convivencia escolar que ellos desarrollan.

\section{Relación entre las formas de administración del conflicto escolar y las formas de convivencia escolar}

Los documentos presentados por el Ministerio de Educación (Mineduc, 2003a, 2003b, 2004, 2011, 2011b, 2013) evidencian la innegable relación entre las formas de administración de conflicto y los estilos de convivencia al interior de una comunidad educativa. El objetivo de la propuesta ministerial es introducir dos mecanismos de integración sistémica en los centros educativos: la normativa escolar y los procedimientos de abordaje pacífico de conflictos (negociación, arbitraje y mediación) (Mineduc, 2002). 
Ahora bien, la Política de CE, publicada el año 2011 ha apoyado nuevas acciones, como la contratación de ATE (Asistencia Técnica Educativa), que externaliza la responsabilidad de la escuela; y la promulgación de la Ley de Violencia Escolar (2011). Este último componente presenta ambigüedad al moverse entre dos paradigmas que tensionan la forma de abordar el conflicto (Autores, 2012). Por una parte, es un paradigma punitivo, que sanciona las agresiones; y por otra, es uno de carácter formativo, que pretende abordar los conflictos de manera pacífica, apuntando al restablecimiento de las relaciones.

Dentro de las prácticas y lineamientos que el Ministerio de Educación propone para la escuela, es posible comenzar a distinguir la concepción de escuela como espacio para la formación ciudadana (Oraisón, \& Pérez, 2006). Por consiguiente, dependiendo del nivel de participación que tengan los sujetos de un establecimiento escolar, como también de los espacios generados para ello; se pueden identificar dos actitudes respecto a la $\mathrm{CE}$. Por una parte, existe una actitud de intransigencia, de tolerancia cero frente a comportamientos indeseados y que se intentan corregir a través de la imposición autoritaria de normas y reglamentos. Una segunda actitud; asume la importancia de la negociación entre los sujetos, orientada a través del conocimiento y respeto de éstos hacia las normas consensuadas con antelación (Tuvilla, 2007).

Por consiguiente, es posible sostener que la forma en que se administre el conflicto se corresponderá con las formas de gestión de la CE (Fierro, 2012; Jares, 1997; Mora, \& Estepa, 2012; Tuvilla, 2007).

Relación con una convivencia autoritaria. La administración tecnocráticapositivista del conflicto se relaciona con el enfoque heterónomo de convivencia escolar. Busca organizar los comportamientos (Halcartegaray, 2009) y la vida de la escuela atendiendo a una proyección de un estado ideal de la realidad (escuela) (Grundy, 1991; Habermas, 1990; Jares, 1997; Prieto, 2001). Por tanto, la calidad de la educación depende de si la escuela cumple con ciertos indicadores externamente.

Este paradigma, no analiza la forma en que los propios agentes crean y mantienen el sistema (Brigido, 2006). El foco estaría puesto en la organización y administración, por consiguiente, el fin de la educación se comprendería como el mantenimiento de un orden estructural y social (Valencia \& Mazuera, 2006).

Milicic y Arón (2000), agregan que la gestión escolar, al ser percibida como autoritaria por los integrantes de un establecimiento, produce la generación de un microclima distinto al percibido de forma general, por lo tanto, pequeños grupos (de profesores o de estudiantes) se protegen del efecto adverso que tiene sobre ellos esta modalidad de gestionar la escuela. Las autoras refieren a climas tóxicos, cuyas características son atribuibles a una gestión de la convivencia autoritaria (Milicic, \& Arón, 2010). Asimismo, los centros educativos que utilizan la sanción como herramienta para abordar los conflictos, buscan erradicar comportamientos indeseados sin ofrecer alternativas de conducta más saludables ni generar un aporte a las necesidades y sentimientos de los involucrados (Vicente de Abad, 2010). Por ende, se observa que, el conflicto es visto siempre de manera negativa y es abordado mediante castigos, persistiendo con la lógica punitiva instaurada (Jares, 1997).

Una investigación de la Unicef (2011) sobre violencia escolar realizada en Latinoamérica puso en evidencia que un $63 \%$ de los estudiantes chilenos apoya la existencia 
de medidas estrictas para resolver aspectos de conflictos y violencia. Además, son los alumnos de familias de bajo nivel educativo que defienden y apoyan la irrupción de medidas más estrictas, por sobre los estudiantes de familias con un alto nivel educativo.

Relación con un estilo de convivencia democrático funcionalista. La perspectiva tecnocrática positivista orientará la escuela hacia un estado ideal propuesto por un tercero. Atendiendo a lo anterior, la Conferencia General ratificada en noviembre de 1995 por la Unesco, titulada "La educación para la paz, los derechos humanos y la democracia" (Unesco, 1995), declara que la educación para la paz consiste en apreciar el valor de la libertad y del ejercicio cívico para trabajar con una comunidad democrática, pacífica y justa.

Posteriormente, la Asamblea General de las Naciones Unidas (ONU, 1999) en su Resolución A/RES/53/243, de 1999, definió los conceptos de "cultura de no violencia" y de "cultura de paz" como un conjunto de valores, que se basan en el respeto a la vida y los derechos humanos, la promoción y la práctica de la no violencia, a través de la cooperación y el diálogo.

Si bien valoramos la declaración de la Unesco, la cultura para la paz abordada tecnocráticamente, generó un nuevo marco de control sobre los comportamientos de los diferentes actores educativos. De esta manera, su instalación queda atrapada en una paradoja; esto es, se promueve la educación para la libertad de los sujetos, sin embargo, se dictamina que deben ser libres, pacíficos y democráticos. Así, la escuela tendrá obligadamente que demostrar que trabaja para la democracia y la libertad (Santos Guerra, 2004).

De acuerdo con Nail Kroyer, Muñoz Reyes y Ansorena Carrasco (2012) y Vicente de Abad (2010), la gestión de una convivencia democrática busca un equilibrio, instalando una disciplina que ejerza control sin ser autoritaria y que fomente la autonomía sin caer en la permisividad. Este aspecto deja entrever la reproducción de una lógica donde el conflicto sigue siendo regulado (Aceituno, Muñoz \& Vásquez, 2012; Santos Guerra, 2004). Sin embargo, de acuerdo a Ianni (2003), la escuela debe ser un lugar significativo para niños y jóvenes y que contribuya a la construcción de su subjetividad. Es, ante todo, un espacio que privilegia la formación humana, y al que se le exige adaptarse a condiciones de convivencia democrática y a parámetros de equidad (Hernández, 2012; Larraín, \& López, 2006; Obando, 2008).

En este sentido, García y de Alba (2007) proponen confiar en las capacidades de participar individual y colectivamente y delegar responsabilidades que trasciendan a un mero simulacro participativo. Educar para la democracia y la gestión de conflictos, supone fomentar la participación ciudadana en relación a la toma de decisiones. Del mismo modo, para Blanco (en Hirmas y Eroles, 2008), Fierro (2013) y Pérez (2009), el fortalecimiento de una convivencia democrática depende del abordaje y modificación de las estructuras vinculadas a la organización escolar, potenciando capacidades reflexivas e integrando la participación de los distintos estamentos en la elaboración y seguimiento de las normativas escolares (Fierro, Carbajal, \& Martínez Parente, 2010).

Administración crítica del conflicto y su relación con una convivencia democrática sociocritica. Desde esta perspectiva se entiende que el conflicto es 
inherente a toda organización social y su ausencia es lo que podríamos considerar como inusual. La investigación nacional, ha constatado que una CE democrática se caracteriza por prácticas inclusivas y por una cultura escolar en la que se acepta la diversidad de sus miembros (Becerra, Tapia, Mansilla, \& García, 2010). De acuerdo con Fierro (2013), el conjunto de prácticas relacionales que se construyen en la convivencia, dan lugar a procesos de inclusión o exclusión, de participación o segregación.

Desde esta perspectiva, estudiantes, profesores, directivos y apoderados son sujetos que pueden actuar a partir de la relación dialéctica que establecen entre el pensamiento y la acción (Ghiso, 1999), transformándose en agentes de cambio educativo, y, por consiguiente, de transformación social.

Esta última forma de comprender el conflicto la podemos encontrar en aquellos contextos educativos en donde prima la ideología sociocrítica (Jares, 1997). No existe un escenario educativo ausente de conflicto; sin embargo, pareciera que las políticas educativas estuvieran planteadas para un contexto homogéneo, en donde el conflicto debe ser "resuelto". Ghiso (1999), plantea que aquellos centros educativos que niegan y evaden el conflicto, no forman para actuar e impiden y controlan el pensamiento crítico y reflexivo de los actores que los conforman.

Una perspectiva crítica de la educación tiene como propósito "transformar la educación a través del cambio de las prácticas educativas, los entendimientos educativos y los valores educativos" tanto de los actores que forman parte del proceso "como de las estructuras sociales e institucionales que definen las posibilidades de acción" (Carr y Kemmis, 1988, en Jares, 1997, p. 20).

\section{Metodología}

Esta investigación adscribe al enfoque cualitativo y su diseño corresponde a un estudio de casos múltiples de tipo microetnográfico. Se describe primordialmente lo que está sucediendo y por qué, con la finalidad de mostrar el perfil de una situación (Martínez Carazo, 2006), es decir, avanza desde el nivel descriptivo al interpretativo. El objetivo final es ofrecer nuevas interpretaciones que permitan establecer relaciones entre la administración del conflicto que una escuela instaura y el tipo de convivencia que de ella se genera.

Para esta investigación, el proceso relevante para investigar es la forma en que los establecimientos descritos administran el conflicto en sus comunidades y cómo estos se ajustan a un estilo autoritario, democrático funcionalista o bien a un estilo democrático sociocrítico de convivencia escolar. De esta manera, se indagó en la realidad específica de cada liceo y de sus rasgos característicos, al ser reconocidas como un evento específico (Hernández-Sampieri, 2003).

\section{Participantes}

Se seleccionó dos liceos municipales ${ }^{8}$, cuyos estudiantes obtuvieron altos puntajes en la prueba estandarizada PISA el año 2009, no seleccionan a sus estudiantes, perte-

8 Los establecimientos fueron escogidos tras obtener los resultados del índice de ambiente escolar arrojado por las escuelas participantes de la fase cuantitativa (año 2012) del proyecto Fonide N11130. 
necen a un Nivel Socioeconómico bajo ${ }^{9}$ y obtuvieron un alto índice de Ambiente Esco$\operatorname{lar}^{10}$ (sobre el percentil 75 de la muestra nacional), es decir poseen un buen Ambiente Escolar (Autores, 2012). En cada una de las escuelas participaron los miembros de la comunidad escolar (director y equipo de gestión; docentes; apoderados; estudiantes; asistentes de la educación y sostenedor).

Asimismo, se consideró de vital importancia los órganos colegiados: consejo de profesores, centro de padres y centro de alumnos. Los participantes fueron seleccionados con técnica de muestreo intencionado, según su potencial para entregar información valiosa en relación al caso. A su vez, se incorporó nuevos actores que permitan profundizar información, sumándose al estudio a través de la técnica "bola de nieve”, o muestreo en avalancha (Quintana, 2006)

Tabla 3

Caracterización de las escuelas

\begin{tabular}{ccc}
\hline Características & Establecimiento A & Establecimiento B \\
\hline Dependencia & Municipal & Municipal \\
Índice de ambiente & 1,65 & 1,60 \\
escolar & 600 & 1039 \\
$\mathrm{~N}^{\circ}$ de estudiantes & Básica $\left(7^{\circ} \mathrm{y} 8^{\circ}\right)$ y Media & Básica $\left(7^{\circ} \mathrm{y} 8^{\circ}\right)$ y Media \\
Nivel de enseñanza & Lenguaje: 506 & Lenguaje: 552 \\
Puntajes Prueba PISA & Matemáticas: 475 & Matemáticas: 527 \\
2009 & Ciencias: 505 & \\
\hline
\end{tabular}

Fuente: Elaboración propia

\section{Técnicas de producción de datos}

Entrevistas activas. El carácter activo de las entrevistas fue dado al compartir con los participantes su clasificación como escuela con alto Índice de Ambiente Escolar en contexto vulnerables, tras lo cual se indagó en sus significaciones y atribuciones a los elementos explicativos de esta situación (Holstein, \& Gubrium, 1995; Denzin, 2001).

Entrevistas activas grupales. Encargado de Convivencia, Dupla Psicosocial, Equipo de Gestión, Miembros del Consejo de Profesores, Miembros del Centro de Padres, y Miembros del Centro de Estudiantes.

\footnotetext{
9 NSE: Se considerará el indicador propuesto por PISA ECSC, índice que se compone de 3 elementos i) posesiones del hogar, posesiones culturales y recursos educativos en el hogar. ii) cantidad de libros del hogar y iii) educación de los padres expresada en años de escolaridad.

10 Para construir el IAE se consideraron las opiniones que dieron los estudiantes a los ítems de las dimensiones de valoración de la escuela, apoyo de profesores y visión positiva. A esto se le sumó las respuestas de los apoderados en las dimensiones de valoración de la escuela y participación. Finalmente, se sumaron las respuestas de los directores a las dimensiones de autonomía, participación y visión positiva. Todos los datos fueron normalizados.
} 
Observaciones participantes. Registros de campo, en los espacios de aula, relación social (entrada y salida del colegio) y recreos. Estuvieron focalizadas en las prácticas de administración de conflictos y del ambiente escolar.

\section{Procedimiento}

Se solicitó la autorización al establecimiento escolar y el consentimiento informado de los participantes. En el caso de los estudiantes, además se solicitó su asentimiento. La producción de datos se llevó a cabo durante una semana en cada establecimiento a través de una microetnografía, donde la producción de información se realiza de manera más breve y concentrada (Atkinson, Donev, \& Tobias, 2007).

\section{Análisis de datos}

Se realizó un análisis interpretativo por escuela (Ibáñez, 1986) bajo la estrategia de Análisis de Contenido (Cáceres, 2003; Franzosi, 2010) avanzando hacia la categorización inclusiva y contextual. De este modo, las dimensiones de análisis guiaron las interpretaciones, sin que impusieran categorías prestablecidas a los datos producidos, adoptando finalmente una aproximación que les "de voz a los datos". De esta forma, la información que se presenta compendia a ambas escuelas por categoría. Con este conjunto de categorías se procedió a realizar un proceso de validación intersubjetiva de manera para mantener aquellas categorías que fueran conceptual y metodológicamente consistentes y "robustas".

\section{Resultados}

\section{Procesos de administración del conflicto}

Es preciso sostener que toda forma de convivencia que se construya a partir de los procesos de administración posee una noción subyacente acerca del conflicto, aspecto que se intenta desentrañar en estos resultados. A continuación, presentamos una serie de subcategorías que conforman este núcleo de sentido, ya desarrollado en el marco de referencia, entre las cuales, destacan los siguientes discursos en convivencia.

Alta normatividad y ambigüedad en la reglamentación. De acuerdo a la producción de datos realizada para esta investigación, en los dos liceos estudiados existen procedimientos predeterminados para resolver los conflictos que en estos susciten. Ambos establecimientos elaboran sus reglamentos siguiendo las premisas de la Política Nacional de Convivencia Escolar del Ministerio de Educación (2011). No obstante, desde el discurso de los estudiantes, se descubre una sensación de arbitrariedad y contrasentido respecto a la aplicación de las normas; se presenta una normatividad excesiva, plasmada con severidad por las autoridades que restringe y paraliza a través de la amenaza a posiciones disidentes.

De acuerdo con Benítes (2011), en muchos establecimientos no existe un modelo claro y explícito de convivencia que oriente las acciones con que los actores de un centro educativo abordan los conflictos. Esto no significa que un reglamento pretenda cubrir la totalidad de situaciones ni que se convierta en un arma a favor de un estamen- 
to por sobre otro. Se trata de evitar la improvisación y desorientación de los actores educativos, formulando normas claras, justas y conocidas por todos (Benbenishty, \& Astor, 2005). La falta de un monitoreo constante y de una planificación coherente con las necesidades de cada centro (Mena, Becerra, \& Castro, 2011), tiene implicancias en el desarrollo de la convivencia escolar, pues se traduce en la construcción de relaciones carentes de confianza y en el debilitamiento del principio formativo y valórico que podría promover la administración del conflicto (Ramírez, 2007). Dicho tratamiento se aproxima a una perspectiva funcional autoritaria de la convivencia, en tanto la normatividad se presenta desprovista de un sentido formativo, plasmando la importancia del orden para el "correcto" funcionamiento de la escuela.

En ambos establecimientos se otorga preponderancia a la reglamentación vigente para resolver una situación complicada,

Mira nosotros somos de 4to y la verdad es que cuando los niños chicos fuman marihuana no les dicen nada y nosotros hacemos algo y nos cancelan todo, pa' qué andamos con cosas si es verdad, debería ser al revés porque nosotros nos vamos, pero si hacemos algo nos suspenden, a los niños chicos le ayudan (Entrevista Estudiante, Liceo A)

Yo considero que tienen que haber normas fijas y a los chicos, como son cosas simples, no deberíamos darles mucha explicación y ellos deberían darse cuenta que deben cumplir con esas cosas. Hay que cumplir con el reglamento pero sin dar tantas explicaciones. (Entrevista profesor Liceo B)

El análisis de las citas refleja que las normas se orientan principalmente a una situación procedimental que no intenta prevenir una situación conflictiva. Esta instancia se transforma en un mero trámite que excede el sentido de la norma. Para ello, según Cornejo y Redondo (2001), las autoridades de los centros educativos acuden a recursos heterogéneos, que en la mayoría de las veces son aplicados de manera particular, en lugar de ser procesados colectivamente, distanciándose inclusive de aquello plasmado en los reglamentos internos.

La clara intención de no dar explicaciones, la rigidez y la preponderancia del papel del profesorado por sobre la voz del estudiantado evoca la construcción de una convivencia altamente normativizada y verticalista, que además degrada la posición de estudiantes, reduciendo su involucramiento en las normas que rigen para ellos. Dichas características vislumbran un tránsito bidireccional entre una convivencia funcional autoritaria y una convivencia funcional democrática. De acuerdo con Perrenoud (1996), la cultura escolar se concentra en las disciplinas que permiten la productividad en una organización, es decir, el conocimiento de la normativa que permite reconocer las restricciones que en la escuela existen, respetar a las autoridades y saber actuar en diferentes circunstancias; características que corresponden a lo que el autor denomina "oficio de alumno"; que permitirá al estudiante comprender las reglas del juego para adaptarse a las "maneras adecuadas" de convivir en la sociedad.

Control y homogeneización. En ambos establecimientos se vislumbra una orientación hacia el orden y el control. Las normas se configuran como elementos construidos únicamente para obedecer y, por consiguiente, lograr el control de todo aquel comportamiento que transgrede lo normado; no así para discutir y replantear su origen, legitimidad e implementación. Dicho aspecto se presenta nítidamente en las siguientes citas: 
Tenemos todo bien controlado. El alumno no puede salir solo, tiene que venir el apoderado a retirarlo, hay un control de retiro, donde sale el curso, la hora, el motivo, el apoderado, nombre, apellido, número de carnet, y si tiene prueba no sale, primero la prueba (Entrevista Profesor Liceo A)

Entre alumnos e inspectores no se llevan tan bien que digamos, porque como ellos se dedican a controlar que los alumnos tengan el uniforme, estén en su clase; como a los alumnos no le gusta eso incluso a veces llegan a contestar y por eso van a la inspectoría y después les hacen llamar al apoderado (Entrevista estudiante Liceo A) El tema de los inspectores fue fundamental, porque yo me acuerdo que cuando llegué había pocos inspectores, uno que otro por aquí por allá, y no había tanta vigilancia de los alumnos. Hay más control de la puerta igual (Entrevista Estudiante, Liceo B) Se puede observar que distintos estamentos exigen control para el funcionamiento ideal de la convivencia escolar. Aspecto que dificulta la aparición de un posicionamiento crítico respecto al conflicto y facilita el convencimiento respecto a que el control acompañado de un alto grado de autoritarismo son los pilares de una buena educación (Carozzo, 2012). Existe una necesidad de que sea otro quien construya una norma que le dicte la vida, desconoce cómo moverse desde otro ámbito. Más fuerza toma el hecho de que el estudiante no cuestione una alternativa para resolver los conflictos (ni menos que pueda ser gestionada colaborativamente), es decir, poder generar un componente democrático en la convivencia escolar.

Otro aspecto relevante, es la especial preocupación que muestran tanto estudiantes como profesores y apoderados por el tratamiento de estos aspectos, abogando por la eliminación de estudiantes que transgredan las normas.

E: ¿Qué hacen cómo inspectoría cuando hay peleas?

P: "Primero llamo a la alumna, después tienen que traer a su apoderado, las peleas son por temas adolescentes, yo le digo a las chiquillas que parecen peleas poblacionales. Ahí se ve con su libreta cómo está el historial y si su falta es leve o no según el manual (Entrevista Inspector Liceo B)

Estas técnicas para abordar las problemáticas que surgen, de una convivencia marcadamente autoritaria, desvirtúan el aspecto formativo de la educación e inducen un rechazo por parte de los estudiantes provocando resentimiento, conformismo y obediencia, conductas que los estudiantes reproducen socialmente y replican en otros ámbitos (Ortega, 2012).

Asimismo, llama la atención que se otorga excesiva importancia al uso de uniforme, característica propia de una convivencia autoritaria de afán homogeneizador, que no se hace cargo ni de las transformaciones relacionales ni de las nuevas subjetividades que circulan en la escuela actual. La escuela se convierte en una escuela como si fuera, como sostiene Molina (2002, en Baeza, 2002), en una Escuela de Apariencia. En las observaciones en los distintos espacios y en las entrevistas realizadas, se expone que el uniforme opera como metáfora de control y dominación. Al ser un foco permanente de situaciones conflictivas, es utilizado para asociar la imagen a una buena convivencia.

Siempre nuestro liceo, entre los municipales ha sido como un liceo que sobresale, porque siempre los niños andan bien uniformados, las alumnas con su uniforme no así muy cortito, el corte de pelo, el zapato, y eso este año está más relajado, y eso lógicamente nos perjudica a nosotros; la imagen del colegio (Apoderado Liceo A) 
(...) incluso consideramos que falta rigidez, falta orden y estabilidad, antes todos peinados con el blazer, el vestón en el caso de los hombres, el jumper, son cosas que antes uno pensaba que no importaba mucho, pero se nota la diferencia en la exigencia (Estudiante Liceo A)

Aspectos punitivos y rol de la autoridad. En ambos establecimientos se advierte el carácter punitivo del abordaje de los conflictos al interior de la comunidad, que se refleja en la necesidad de contar, no sólo con procedimientos, sino además con distintas formas de sanción, que van desde la derivación a inspectoría hasta la suspensión, o en última instancia, a la expulsión del estudiante. Las prácticas y los discursos que sugieren los distintos actores de los centros educativos, apuntan a un abordaje del conflicto que se enmarca dentro de una convivencia escolar funcional autoritaria, ya que las sanciones se ligan a un patrón burocrático, cuyo sentido no es más que el de erradicar el conflicto a través de prácticas que constriñen las posibilidades de acción y crítica de los estudiantes, fortaleciendo formas de relación verticalistas y funcionales, que fracturan la premisa formativa para alcanzar primero el desarrollo humano y después el académico (Carozzo, 2012). Por tanto, la convivencia que se está construyendo oprime toda voz y conducta disidente, lo que implica, por lo demás, que sea un grupo reducido quienes determinen qué comportamiento son deseables y cuáles no. Si bien existe un enfoque formativo por parte de algunos actores, el predominio del enfoque punitivo se hace evidente en el discurso tanto de estudiantes, profesores, paradocentes y apoderados, existiendo congruencia entre los actores mencionados en relación a la necesidad de un enfoque individual y de eliminación del conflicto. Se puede observar, en la siguiente cita, la nula efectividad que poseen las prácticas punitivas, puesto que revelan un aumento del problema.

Se sorprendieron a muchos chicos fumando marihuana en el baño, ahí llegamos a hacer vigilia, a los chicos se les llamó a los papás y se les suspendió, fueron caducadas sus matrículas. Hicimos seguimiento, con los inspectores, con el centro de padres y con la PDI, se cortó. Volvió de nuevo y volvió con fuerza (Entrevista apoderado/a Liceo A)

Respecto al rol que asume la autoridad, en el Liceo B, el autoritarismo se encarna en la figura omnipresente y controladora de la directora, quien reprime la intención de los estudiantes por construir una mejor escuela.

Para cuándo fue la toma del liceo, llamó uno por uno a los líderes y les dijo que si se tomaban el liceo ella (directora) los iba a echar, entonces que más nos espera a nosotros decirle algo (Entrevista Estudiante, Liceo B).

Eso es lo bueno que tiene ella, (la directora) si los chicos se portan mal, los castigan, buenos castigos les dan. No se po', hay chicos que se han tenido que ir (...) los cambian de curso también (Apoderado Liceo B).

De esta manera, la noción del conflicto como elemento negativo, fortalece un estilo de convivencia autoritaria. En esta cita se presenta la figura de la amenaza para evitar un comportamiento indeseable para algunos. Así, la instancia sancionadora evita un comportamiento y además inhibe a que otros participen de una forma similar, es decir actúa utilizando el miedo como instrumento de disuasión. Asimismo, se muestra como una opción viable la expulsión de los estudiantes, lo que implica deshacerse de un elemento perturbador para que sea otro quien se haga cargo.

En el Liceo A hallamos diálogos similares respecto al Liceo B. La diferencia está en la figura del director que dejó su cargo y que es reemplazado. Se aprecia una 
añoranza de autoridad y disciplina, manifestada a través del anhelo de una lógica patriarcal y punitiva.

Los colegas dicen que el nuevo director no tiene la misma forma; de que sale al patio, grita y los niños tienen miedo, este director tiene otro estilo de administrar, (...) entonces todos lo ven como incompetente, no lo entienden. (Entrevista Profesor, Liceo A)

Benítes (2011), plantea que el modelo punitivo es aquel que predomina en los centros educativos cuyas acciones se fundamentan en la reparación del daño a través del castigo. Pese a lo anterior, como se demuestra en las siguientes citas, nos encontramos con actores educativos que intentan revertir el carácter punitivo de las sanciones que implementan otros miembros del centro educativo. Se manifiesta una resistencia de los profesores al tipo de CE que se está construyendo.

Nos ven siempre como "este que coloca las reglas, el demasiado punitivo" y cuando llegaban a la oficina les dábamos otro trato, un trato más empático, y tratándoles de hacer entender que toda falta va de la mano de una sanción, y la sanción tiene que ser de tal forma, gradual a esa falta (Entrevista Profesor Liceo B)

(..) Si yo tengo un problema conductual en la sala de clases yo lo que hago con el chico es conversar con él, qué le pasa, por qué él está así de esa forma y después si sigue igual ahí lo derivo a las instancias correspondientes. Pero hay muchos profesores que pa' afuera al tiro, y a la segunda y a la tercera y siguen echando pa' fuera" (Entrevista Profesor Liceo A)

El aspecto relevante que genera este exceso de sanciones en los centros, es la connotación positiva que estudiantes y apoderados otorgan a la disciplina castigadora (Halcartegaray, 2009). En esta misma línea, una investigación realizada por Cornejo y Redondo (2001) en Liceos de la Región Metropolitana, suma evidencias respecto a que las normas y sus sentidos no son internalizadas por los estudiantes desde un marco formativo, sino que a partir de la sanción, elemento regulador que los afecta de manera directa. Según los autores, existe una tendencia por parte de los estudiantes a regular su comportamiento mediante castigos que autoridades administran, en perjuicio de una relación colaborativa y una visión formativa del conflicto. Respecto a esto, los establecimientos estudiados, no difieren de las prácticas de los establecimientos de la investigación mencionada. En ambos establecimientos prima una lógica punitiva, no obstante en el establecimiento A, se vislumbra una tensión entre quienes respaldan la sanción y el castigo, y aquellos que optan por hacer del conflicto una instancia formativa.

Yo creo que suspenderlos es peor. Yo creo que hay que tomarlos, insertarlos en un centro. La solución no es echarlos (Entrevista apoderado/a Liceo A)

$\mathrm{Y}$ yo le digo a los colegas, porque yo no soy ese tipo de personas que suspensión, suspensión, primero busco por qué motivo el niño no tiene empatía con el profesor, hablo con la profesora (Entrevista Inspector/a Liceo A)

Existe un intento de algunos de los actores pertenecientes al Liceo A, de evitar la sanción e intentar posicionarse desde una lógica formativa; una visión que se acerca a la búsqueda de un tratamiento social del conflicto. En el Liceo B también existe un intento de evitar la sanción, sin embargo, no hay un esfuerzo por formar a partir del conflicto, el carácter punitivo se encuentra naturalizado tanto en el discurso como en las prácticas de los distintos actores. Tal como demuestran las siguientes citas: 
El atraso aquí es enorme, más de 100, 150 alumnos atrasados, yo podría cerrar la puerta a las 8 y se van para la casa, pero no se puede porque el Ministerio dice que no se puede enviar a nadie para la casa (...) esto repercute en las clases y se genera otro problema (Entrevista Inspector/a, Liceo B)

Este aspecto, lo podemos asociar a una convivencia democrática funcionalista, puesto que devela cómo el poder de un organismo ata de manos a quienes tienen la obligación de formar y genera un choque en las prioridades del centro educativo.

Yo soy reacia a las suspensiones, yo digo mejor es tenerlos en el liceo y que hagan algo, que limpien las murallas, o algo, si a nadie se le va a caer nada porque hagan actividades de limpieza o mantenimiento dentro del liceo (Entrevista apoderado/a Liceo B)

En este aspecto, observamos cómo en el establecimiento B surge la creencia de que se puede suplir una sanción tradicional por una que aporte a las necesidades del centro educacional, como por ejemplo la infraestructura. En este caso, la noción de conflicto como algo negativo permanece y a pesar de las buenas intenciones no existe una orientación formativa que apunte hacia un valor democrático.

En contraposición a esta visión del conflicto como algo negativo e indeseable, surge el abordaje del conflicto desde una perspectiva sociocrítica. Esta perspectiva plantea que el conflicto constituye una oportunidad para visibilizar temas que no han sido tratados y, de esta manera, crear nuevas formas de relación. Siguiendo a Llaña (2011, p. 55) "el conflicto destruye y al mismo tiempo crea, divide y al mismo tiempo unifica", constituyendo un poderoso factor de consolidación grupal. Desde esta perspectiva, si el conflicto es abordado social y democráticamente permite, por parte de todos los actores de la comunidad educativa, la reflexión sobre el propio funcionamiento del centro educativo, y por consiguiente, la creación colectiva de nuevas soluciones.

Baja participación (pseudoparticipación). A pesar de que docentes y directivos instalan en sus discursos una administración del conflicto gestionada democráticamente, son sus propias palabras, además de lo señalado por estudiantes, las que evidencian contradicciones entre los principios democráticos que emergen desde las políticas públicas y las prácticas de ambos establecimientos (Aceituno, Muñoz, \& Vásquez, 2012; Santos Guerra, 2004). En ambos establecimientos, la concepción de lo democrático se remite a la posibilidad de elegir cuerpos representativos con un poder de decisión limitado. En la siguiente cita los estudiantes valoran que hayan podido ser ellos quienes elijan a un centro de estudiantes, sin embargo, no posicionan como un conflicto el hecho de que hayan sido obligados, en el pasado, a conformar dicho cuerpo representativo. En este sentido, podemos referirnos a una pseudoparticipación puesto que se forman estas entidades como parte de requisitos que el establecimiento debe cumplir.

Por ejemplo ahora se escogió un centro de alumnos, se hizo una votación democrática, no fue como el año pasado que los profesores prácticamente escogían a los integrantes del CEAL (Entrevista Estudiante Liceo A)

Nosotros tenemos reunión una vez al mes con las directivas, el problema es que se supone que nosotros organizamos la reunión y la directora aparece y ella hace la reunión. Ella habla sus cosas y por ejemplo nos deja los últimos diez minutos para salir a recreo (Entrevista Estudiante CEAL, Liceo B) 
Al analizar las citas es evidente que, en ambos casos, los estudiantes son elegidos y son invitados a participar en propuestas diseñadas por otros estamentos (Obando, 2008), no como una participación real; puesto que para ello, según García y De Alba (2008), la educación debiese estar vinculada a una participación activa, comprometida y desarrollada a través de la acción sobre problemáticas dentro del entorno escolar, donde el estudiantado tiene una capacidad real de intervenir y ser escuchado. Aquí cobra relevancia lo planteado por Fierro, Carbajal y Martínez Parente (2010), quienes sostienen que las estructuras con que se ha concebido y diseñado la participación estudiantil presentarían dificultades a la hora de implementar un modelo de convivencia participativo y democrático. En este sentido, se presenta una convivencia aparentemente democrática; puesto que los estudiantes adoptan un rol pasivo respecto a situaciones críticas, operando meramente para solucionar aspectos técnicos sugeridos verticalmente. De esta forma, el estudiantado contribuye a la invisibilización del conflicto y la perpetuación de una convivencia con fuertes rasgos autoritarios.

Nos encargamos de hacer las cosas para el aniversario, vemos cuando los niños tienen problemas, ahora queremos hacer un día cultural, estamos en un proyecto de banda, estamos haciendo en conjunto con dirección algo que nos aquejaba, esas cosas como jaboneras para la higiene, confort. Nosotros queremos mejorar el liceo, queremos mejorar el establecimiento, entonces son cosas pequeñas pero que les sirven a los niños (Entrevista Estudiante CEAL, Liceo A)

Con todo esto del paro nosotros hablamos para tener más autonomía como CEAL, pero nunca resulta porque no existen los espacios. O sea, nosotros no podemos hablarle de cosas así a la directora, no existe como esa confianza". (Entrevista Estudiante CEAL, Liceo B)

En las siguientes citas se refleja la nula participación que tanto estudiantes como apoderados tienen en la construcción de los reglamentos que administra el conflicto, aspecto que devela un tratamiento individual protagonizado únicamente por un estamento:

El año pasado cambiaron el manual de convivencia, ellos lo cambiaron y después dijeron que todos los alumnos tenían que firmar para saber que lo cambiaron. Pero el papel decía que todos estaban de acuerdo con lo que decía y yo me acuerdo que mi curso no quiso firmar y fueron como tres profesores prácticamente a obligarnos a firmar, nos retaron incluso porque no habíamos firmado. (Entrevista estudiante Liceo B).

Nosotros no tenemos ni arte ni parte en los reglamentos del liceo, tenemos que firmar no más y firmamos, no nos queda otra, el año pasado hubo modificaciones por ejemplo y no informaron (Entrevista apoderado Liceo B).

Esta relación de autoridad-sumisión, obstaculiza aún más las posibilidades de participar, en tanto el estudiante es convertido en un ser pasivo y receptor de la realidad dada, por tanto, se construye una convivencia que se remite a interiorizar dicho proceso de sometimiento a todo elemento autoritario existente (Barroso y Collado, 2012).

Enfoque experto. En estos centros no existe un proyecto cooperativo cuya dirección sea una transformación óptima de la realidad que produzca la humanización de la convivencia (Gijón, \& Puig Rovira, 2010). Esta condición facilita la aparición de otras figuras relevantes para los procedimientos de estos establecimientos, tales como inspectores y profesionales especializados. La siguiente cita evidencia la aparición de más inspectores para controlar la emergencia de un comportamiento indeseable, con el fin de eliminarlo. 
Al principio cuando llegaron personas nuevas, con nuevas influencias, llegaron con principios distintos a los que tenía el liceo, venían con otras expectativas, no a estudiar, venían a chacotear (...) llamaron más inspectores, y (a los alumnos nuevos) los eliminaron del liceo, ya no están (Entrevista estudiante Liceo A)

En las prácticas de ambos establecimientos, es relevante el protagonismo que se entrega a terceros expertos para solucionar problemáticas relacionadas con la convivencia; por ejemplo: orientadores, psicólogos, asistentes sociales; quienes en determinadas situaciones asumen el rol de Encargado de Convivencia. Esta figura resulta paradojal, puesto que las políticas ministeriales describen la convivencia como un constructo de carácter social, democrático y participativo, sin embargo, la realidad demuestra que el responsable de la convivencia es solo una persona. Las siguientes citas, vislumbran el poder otorgado a profesionales externos ante una situación de conflicto. En ellas, el conflicto es comprendido como un elemento negativo que requiere ser solucionado por otro.

El año pasado tuvimos que hacer una especie como de cirugía, tuvo que venir una empresa externa a ayudarnos en el tema de convivencia. Vino un psicólogo para trabajar con todos los estamentos (Entrevista Directivo Liceo B).

Entonces, qué hacen con esos niños, primero los mandan con la psicóloga o asistente social y ven lo que está pasando y si esa persona tiene remedio o no, lo dejan o lo expulsan, supuestamente por lo que el director nos contó ya han echado como a cien niños (Entrevista profesor Liceo A).

Estamos frente a la construcción de una convivencia funcional democrática en cuanto la aparición de estos profesionales también responde a los requerimientos que impone el ministerio de educación, pero a su vez, perpetúan un mecanismo que sigue amparando la eliminación de aquello considerado indeseable. En consecuencia, existe el continuo entre una convivencia funcionalista autoritaria y una convivencia funcionalista democrática.

El análisis de estas citas, evidencia que el docente está en un escenario donde ya no tiene la necesidad de lidiar con un conflicto. Los responsables de abordar el conflicto se ubican fuera de las aulas; esto genera una tensión porque los centros educativos cuentan con más profesionales de apoyo para la convivencia escolar, pero produce que el profesor pierda protagonismo respecto al conflicto, limitando su accionar a la transmisión de conocimientos (Giroux, 1990). Al contrario de lo planteado por Alzate (2003; en Tuvilla, 2007) y Leiva (2008), quienes sostienen que los docentes deben ser los encargados de promover el diálogo y la escucha activa que incentiven al estudiantado a explorar sus ideas y sentimientos, por ende, un facilitador que permita a los estudiantes obtener aprendizajes a partir de la experiencia del conflicto. Aquí se pierde el sentido transversal y formativo que el Ministerio pretende instalar (se evita el traspaso de una democracia funcional a una democracia sociocrítica).

Administración reactiva de la convivencia. El tratamiento preventivo, de abordaje formativo y social es escaso en ambos establecimientos. La mayor parte de las acciones para abordar el conflicto son reactivas a diferentes situaciones donde los esfuerzos y la intención por mejorar las prácticas en torno a la convivencia escolar se alejan de procedimientos sistemáticos y eficientes en tanto, no obedecen a un diagnóstico e intervención formal que permita abordar el conflicto de manera sistemática, colectiva y transversal dentro de la escuela (González, 2006). Respecto a los 
principales resultados, es posible afirmar que los docentes y estudiantes no tienen criterios claros sobre su actuar dentro de la escuela, no hay un claro conocimiento de los valores que representan las normas de participación y el compartir con otros. El otro día fui víctima de un motín, los alumnos no entraron a clases porque según ellos yo no soy bueno metodológicamente (...). Ante la carencia total de alumnos, avise al inspector y luego el tema se fue a dirección, yo no fui a la reunión porque no abandoné mi lugar de trabajo y permanecí en la sala durante toda la hora (Entrevista Profesor, Liceo B)

En situaciones como la descrita, que pareciera ser extrema, el conflicto adquiere un tratamiento reactivo y no es utilizado como herramienta para la prevención. El docente no se cuestiona cuál fue el motivo del motín ni la metodología empleada. No existe lugar para cuestionar la propia práctica ni la posibilidad para transformar la relación con sus estudiantes.

Ahí hubo un problema de actitud, que no hizo caso en reiteradas veces de lo que se le estaba diciendo y hubo que mandarlo a inspectoría y ahí se llamó al apoderado y él solucionó el problema. En mi curso por lo menos pasa una vez a la semana que va alguien (Entrevista Profesor, Liceo A)

En ambas citas se observa un tratamiento reactivo del conflicto mediante un procedimiento ineficiente. Mena, Becerra y Castro, (2011) proponen que, existe evidencia que los actores educativos poseen creencias donde la administración del conflicto que orienta la gestión de la convivencia surge sólo de manera reactiva (Mena, Becerra, \& Castro, 2011). En el discurso de los profesores de ambos establecimientos se observa una situación que no pudo ser prevista y su tratamiento, claramente reactivo, posee además un elemento improvisador puesto que, tal como refleja la cita del Liceo B, no todas las situaciones de conflictos son contempladas por la reglamentación vigente. Por lo tanto, el tratamiento del conflicto debe responder a un trabajo elaborado desde la prevención (Tuvilla, 2007). De esta forma, podemos determinar una clara diferencia entre “¿qué hacer cuando ocurre un motín?” a preguntarnos “¿por qué ocurrió ese motín?" o bien, a partir de esta situación, qué aprendizajes y oportunidades de mejora pueden brotar desde una mirada crítica del conflicto. Según el autor, los conflictos no deberían ser tratados desde un enfoque restringido y parcial, sino desde un enfoque ampliado a partir de modelos preventivos e integrados, cuyos pilares se sostengan en una perspectiva global y sistémica.

Abordaje social y autónomo de la convivencia. Pese a la gestión autoritaria presente en los procedimientos de ambos establecimientos, en los discursos del Liceo A surge una característica que no se encuentra presente en el discurso de los actores del Liceo B. En el primero, descubrimos que la forma en que el estamento estudiantil busca resolver los conflictos adopta una postura más activa y autónoma. En este sentido, desde el discurso de los estudiantes, existe un abordaje social caracterizado por la búsqueda de alternativas que son tomadas en conjunto para generar acuerdos. Al contrario de la visión individual presente en las prácticas y discursos de profesores, paradocentes y apoderados.

Hay niños que se pelean de repente acá en la sala, a combo limpio. Nosotros los más grandes, los separamos, los controlamos, las cosas se arreglan en el curso y en el curso se quedan. Nosotros decimos que no tiene que haber bullying, obviamente que echamos la talla pero no así bromas pesadas, no problemas mayores (Entrevista estudiante Liceo A) 
Siguiendo a Delval (2012), los estudiantes debiesen participar activamente en el funcionamiento tanto del aula como de la escuela. Si contribuyen a la administración del conflicto y toman un rol activo en el centro educacional, entonces se facilitará la capacidad de reflexionar y participar en los procesos que se desarrollan en la sociedad más amplia, avanzando desde una convivencia democrática funcionalista hacia una convivencia democrática sociocrítica.

\section{Discusión}

El discurso de los actores educativos que se hace visible en las dos escuelas estudiadas sobre la forma de administración del conflicto, se orienta y alinea con una convivencia de estilo autoritaria. En los centros educativos, el conflicto es abordado desde un enfoque punitivo y reactivo, relativo a un estado de cosas diseñado por las autoridades, pseudoparticipativo y orientado a lo disciplinar, con tratamiento ambiguo de la norma. Se pierden los espacios de creación que impiden que la escuela se invente a sí misma, más bien, tiene el imperativo de mantenerse constante. Todo lo anterior no significa que la única voz de la comunidad educativa presente una orientación hacia una administración funcional autoritaria del conflicto; la diversidad de voces presente en la escuela, permite encontrar discursos que van en la línea de una comprensión funcional democrática del conflicto. Incluso, algunos actores -especialmente los estudiantes- presentan comprensiones en la línea sociocrítica. Desde ña vertiente funcional autoritaria los estudiantes son considerados agentes pasivos (Mena, Becerra, \& Castro, 2011). A este tipo de convivencia se asocian prácticas, en las cuales el conflicto es comprendido como un elemento perturbador que debe ser eliminado para el surgimiento de una buena convivencia escolar.

La diversidad, elemento propio de nuestra sociedad y de toda organización, nos muestra la multiplicidad de voces y posiciones que adoptan los actores de los establecimientos estudiados. En esta investigación, se observa que en los distintos estamentos existe una valoración positiva hacia aspectos castigadores como única vía para la erradicación de conductas inadecuadas y situaciones que son comprendidas como conflictivas.

Existe una interpretación negativa del conflicto y una naturalización de los procedimientos punitivos que regulan las interacciones entre y al interior de los distintos estamentos. El aspecto sorpresivo, es que este autoritarismo se valida y perpetúa a través del discurso de estudiantes, profesores, apoderados y directivos; no se presenta como un factor impuesto desde un lado más fuerte hacia otro más débil, sino que surge como un requerimiento para la buena convivencia. En este sentido, la administración del conflicto en ambas escuelas pareciera transformar la convivencia escolar en una suerte de irradiación del ejercicio de autoridad, que atraviesa gran parte de las prácticas y formas de relación entre y dentro de los distintos estamentos. En ambos establecimientos, el concepto recién explicado se canaliza mediante la figura del Director, en el establecimiento B es una figura omnipotente; y en el establecimiento A es una figura ausente que se extraña y se demanda. Esto, nos invita a reflexionar en torno a una visión paternalista de la educación que busca reforzar la dependencia 
e infantilización del estudiantado, instalando otro espacio que coarta el sentido de reflexión, crítica y empoderamiento de los sujetos (Dabas, 1993).

En ambos establecimientos, se observa una carencia de aprendizaje de las situaciones de conflicto, donde las sugerencias se implementan tarde, son nuevas y obedecen a una improvisación que genera desorientación. En este aspecto, es preciso problematizar el rol del profesor en la organización, puesto que su figura se enfrenta cotidianamente a situaciones de conflicto. Esta investigación descubre la importancia que los centros educativos entregan a agentes externos a la realidad del aula (psicólogos, orientadores, asistentes sociales e inspectores) derivando el conflicto con el objetivo de ser erradicado de la sala de clases. En consecuencia, el docente es relegado de esta mancomunión, asumiendo un rol técnico-reproductor de escasa autonomía, ubicándose en la categoría de un empleado administrativo que acata instrucciones (Núñez, 2004; Prieto, 2001). Estas medidas muestran la escasa confianza en la capacidad de los docentes para ejercer un liderazgo intelectual y moral (Giroux, 1990). En palabras de Ianni (2003), el profesor rinde culto a la eficacia y a la productividad, perdiendo autoridad para ser un agente transformador de la convivencia.

Desde esta óptica, surge la necesidad de defender la escuela como un espacio donde se geste una convivencia democrática sociocrítica, y comprender el rol de los profesores como intelectuales transformativos (Giroux, 1990) que integren el conflicto en la reflexión y la práctica académica en miras a educar ciudadanos capaces de hacerse cargo de sus propias necesidades, intereses y problemáticas para el desarrollo de una mayor autonomía.

Finalmente, otro punto de discusión de esta investigación es la incoherencia manifiesta entre lo que propone el Mineduc y la realidad observada en ambos establecimientos. Los resultados sostienen que este problema se relaciona al tipo de subjetividad estudiantil que indirectamente la política educativa y las prácticas escolares construye (Autores, 2012). Por una parte, se exige a la escuela que todos los miembros de la comunidad participen de su construcción; y por la otra, se otorga el poder y el control al equipo directivo y estamento docente, lo cual resulta contradictorio (Autores, 2009).

Por consiguiente, la normatividad con sus respectivos requisitos se transforman en una "lista de verificación", que se cumple, se respeta, pero no es significativa ni transformadora. La administración del conflicto se limita a la participación simulada de estamentos (García, \& de Alba, 2007), como el estudiantil, que bordea el cumplimiento legítimo de los requisitos ministeriales, mezclando lo denominado democrático con componentes autoritarios; en este sentido, lo más democrático no es sinónimo de menos autoritario, puesto que se obra en función de favorecer al establecimiento de turno. Este concepto de Convivencia (Funcional) Democrática respecto a la administración del conflicto, se hace cargo de la reparación, es decir, volver a una situación al mismo estado que se encontraba antes (Vicente de Abad, 2010), imposibilitando nuevas formas de relación y transformación.

Si bien existe una intención de llevar a la práctica los postulados democráticos (Leiva, 2008), en las realidades estudiadas existe una mezcla de componentes que transitan desde una perspectiva autoritaria hacia lo democrático, donde el conflicto es regulado permanentemente. 
Es necesario avanzar hacia nuevas formas de relación que encarnen los intereses de los propios actores que conforman la comunidad educativa y que consideren su diversidad, y no aquellos que representen los intereses de un sector determinado de la sociedad y de la escuela que busca reproducir relaciones de dominación y desigualdad social. Es una subjetividad que rebasa los límites del espacio escolar y construye una forma de ser ciudadano y, por ende, de convivir con el otro (Borrero, 2005, en Palma, 2011; Giroux \& McLaren, 1998; Perrenoud, 1996), de ahí su relevancia.

\section{Referencias}

Aceituno, D., Muñoz, C., \& Vásquez, G. (2012). Enseñanza y aprendizaje de la participación ciudadana en Chile: Un estudio sobre alumnos y profesores de Historia de $2^{\circ}$ año de enseñanza media. En Nicolás de Alba, Francisco García, \& Antoni Santisteban (eds.), Educar para la participación ciudadana en la enseñanza de las ciencias sociales, Vol. II (pp. 165-177). Sevilla, España, Editora Sevilla.

Acosta, A. (2007). Material para la mejora de la convivencia escolar: Normas de Convivencia. Recuperado de: https://convivencia.wordpress.com/category/2protocolos/p-guias-habilidades/

Autores (2009).

Apple, M. W., \& Beane, J. A. (1997). Escuelas democráticas. Madrid: España: Morata.

Arnal, J. (1992). Investigación educativa. Fundamentos y metodología. Barcelona, España: Labor.

Atkinson, A. C., Donev, A. N., \& Tobias, R. D. (2007). Optimum experimental designs, with SAS. New York, NY: Oxford University Press.

Baeza, J. (2002) Leer desde los alumnos (as), condición necesaria para una convivencia escolar democrática. En UNESCO (Eds.), Educación secundaria: un camino para el desarrollo humano (pp. 163-184).

Banz, C. (2008). Convivencia Escolar. Documento Valoras UC. Recuperado del sitio de internet Educar Chile, http://ww2.educarchile.cl/UserFiles/P0001/File/convivencia_escolar.pdf

Barroso, J. M., \& Collado, J. (2012). La creación de materiales curriculares para promover la participación ciudadana en un sistema educativo coherente. Educar para la participación ciudadana en la enseñanza de las Ciencias Sociales. Vol. I. Sevilla, España: Díada Editora.

Becerra, S., Tapia, C., Mansilla, J., \& García, R. (2010). Las prácticas de convivencia escolar y su impacto en el proceso identitario de los jóvenes en contexto de pobreza. Manuscrito enviado para su publicación.

Benbenishty, R., \& Astor, R. A. (2005). School violence in context: culture, neighborhood, family, school and gender. New York, NY: Oxford University Press.

Benítes, L (2011). Convivencia escolar y calidad educativa. Lima, Perú: Escuela Profesional de Psicología.

Brigido, A. (2006). Sociología de la educación. Bienos Aires, Argentina: Editorial Brujas. 
Cáceres, P. (2003). Análisis cualitativo de contenido: Una alternativa metodológica alcanzable. Psicoperspectivas, 2(1), 53-82.

Carozzo, J. (2012). El bullying en la escuela: Interrogantes y reflexiones. En L. Benítez, J. Carozzo, V. Horna, L. Palomino, C. Salgado, C. Uribe, \& L. Zapata, Bullying y convivencia en la escuela. aspectos conceptuales, aplicativos y de investigación. Observatorio sobre violencia y convivencia en la escuela. Lima, Perú: Observatorio sobre Violencia y Convivencia en la Escuela.

Carr, W., \& Kemmis, S. (1988). Teoría crítica de la enseñanza. Buenos Aires, Argentina: Martínez Roca.

Autores (2012).

Cornejo, R., \& Redondo, M. J. (2001). El clima escolar percibido por los alumnos de enseñanza media. Última Década, 15, 11 -52.

Dabas, E. (1993). Red de redes: Las prácticas de intervención en redes sociales. Buenos Aires, Argentina: Paidós.

Delval, J. (2012). Ciudadanía y escuela: El aprendizaje de la participación. En N. Alba, F. García, \& A. Santisteban (eds.), Educar para la participación ciudadana en la enseñanza de las ciencias sociales, Vol. II. Sevilla, España: Diada.

Denzin, N. (2001). The reflexive interview and a performative social science. Qualitative Research, 1(1), 23-46.

Fierro C., Carbajal, P., \& Martínez-Parente, R. (2010). Ojos que sí ven: Casos para reflexionar sobre la convivencia en la escuela. México, D.F.: SM.

Fierro, M. C. (2013). Convivencia inclusiva y democrática. Una perspectiva para gestionar la seguridad escolar. Revista Electrónica Sinéctica, 40, 1-18.

Franzosi, R. P. (2010). Content analysis. En M. Hardy, \& A. Bryman (eds.), The handbook of data analysis. London, UK: Sage.

García, F., \& De Alba, N. (2007). La educación para la participación ciudadana entre dos polos: el simulacro escolar y el compromiso social. En N. De Alba, F. F. García, \& A. Santisteban (coords), Educar para la participación ciudadana en la enseñanza de las Ciencias Sociales (pp. 297-306). Lugar?: Asociación Universitaria de Profesorado de Didáctica de las Ciencias Sociales.

García, F., \& De Alba, N. (2008). ¿Puede la escuela del siglo XXI educar a los ciudadanos y ciudadanas del siglo XXI? Diez años de cambios en el Mundo, en la Geografía y en las Ciencias Sociales, 1999-2008. Actas del X Coloquio Internacional de Geocrítica, Universidad de Barcelona, 26-30. En http://www.ub.es/ geocrit/-xcol/394.htm

Ghiso, A. (1999). Pedagogía y conflicto: Pistas para deconstruir mitos y desarrollar propuestas de convivencia escolar. Signo y Pensamiento, 34(18), 35-58.

Gento, S. (1994). Participación en la gestión educativa. Madrid: Santillana.

Gijón Casares, M., \& Puig Rovira, J. (2010). Encuentros y convivencia escolar. Educação: Revista do Centro de Educação, 35(3), 367-379.

Giroux, H. (1990): Los profesores como intelectuales: Barcelona, España: Paidós. 
Giroux, H. A., \& McLaren, P. (1998). Sociedad, cultura y educación. Madrid, España: Miño y Dávila.

González, L. (2006). Gestión y convivencia democrática en la escuela. Lugar: Ediciones: OEI.

Grundy, S. (1991). Producto o praxis del currículum. Madrid: Morata.

Habermas, J. (1990). Conocimiento e interés. Madrid: Taurus.

Halcartegaray, M. A. (2009). Análisis y reflexiones sobre reglamentos de Convivencia Escolar: Los supuestos, opciones y visiones a la hora de formular un reglamento. Ficha 1 Valoras UC.

Hernández, C. (2012). Ciudadanía, diversidad y participación: Educar para la participación desde la diversidad. Revista Educação e Políticas em Debate 1(1). Recuperado de: https://www.researchgate.net/publication/236758167_Ciudadania_Diversidad_y_Participacion_Educar_para_la_Participacion_desde_la_Diversidad_Citizenship_Diversity_and_Participation_Education_for_Diversity_ from_Participation

Hernández Sampieri, R. (2003). Metodología de la investigación. 3a. ed. México, D. F.: McGraw-Hill.

Hirmas, C., \& Eroles, D. (coords.) (2008). Convivencia democrática, inclusión y cultura de paz: Lecciones desde la práctica educativa innovadora en América Latina. Santiago, Chile: Unesco-Red Innovemos.

Holstein, J., \& Gubrium, J. (1995). The active interview. London, UK: Sage.

Ianni, N. (2003). La convivencia escolar: una tarea necesaria, posible y compleja. Monografías virtuales, 2 : Ciudadanía, democracia y valores en sociedades plurales.

Ibáñez, J. (1986). Más allá de la sociología. Madrid: España: Siglo XXI.

Jares, X. (1997). El lugar del conflicto en la organización escolar. Revista Iberoamericana de Educación, 15.

Kröyer, O. N., Muñoz Reyes, M., \& Ansorena Carrasco, N. (2012). Normativa y reglamentos de convivencia escolar, ¿Una oportunidad o una carga para la escuela? Educere, 16(55), 373-384.

Larraín, R. \& López, R. (2006). Reflexiones sobre convivencia y democracia. En Juan Ruz (ed.), Convivencia y calidad de la educación. Santiago, Chile: OEI.

Leiva, J. (2008). Interculturalidad, gestión de la convivencia y diversidad cultural en la escuela: Un estudio de las actitudes del profesorado. Revista Iberoamericana de Educación, 46/2.

Llaña, M. (2011). La convivencia en los espacios escolares. Santiago, Chile:.Universidad de Chile.

Autores (2012).

ONU (1999). Declaración y programa de acción sobre una cultura de paz. (A/ RES/53/243), 6 de octubre de 1999. http://www3.unesco.org/iycp/kits/sp_res243. $\mathrm{pdf}$ 
Magendzo, A., Toledo, M., \& Gutiérrez , V. (2012). Descripción y análisis de la Ley sobre Violencia Escolar ( $\left.{ }^{\circ} 20.536\right)$ : Dos paradigmas antagónicos. Estudios Pedagógicos, 39(1), 377-391.

Maldonado, H. (2004). Convivencia escolar ensayos y experiencias. Buenos Aires, Argentina: Lugar Editorial.

Martínez, J. B. (1998). La voz del alumnado: Ausencia temporal de la ciudadanía. Cuadernos de Pedagogía, 275, 56- 65.

Martínez Carazo, P. (2006). El método de estudio de casos: Estrategia metodológica de la investigación científica pensamiento y gestión. Revista de la División de Ciencias Administrativas de la Universidad del Norte, 20, 165-193.

Mena, I., Becerra, S., \& Castro, P. (2011). Gestión de la convivencia escolar en Chile: Problemáticas, anhelos y desafíos. En J. Catalán (ed.), Psicología Educacional: Proponiendo rumbos, problemáticas y aportaciones (pp. 81-112). La Serena, Chile: Universidad de La Serena.

Milicic, N., \& Arón, A. M. (2011). Climas sociales tóxicos y climas sociales nutritivos para el desarrollo personal en el contexto escolar. Psykhe, 9(2).

Mineduc. (2002). Política nacional de convivencia escolar. Hacia una educación de calidad para todos. Recuperado de: http://www.mineduc.cl/wp-content/uploads/ sites/19/2015/12/politica-noviembre-definitiva.pdf

Mineduc. (2004). Política de participación de padres, madres y apoderados/as en el sistema educativo. Recuperado de: http://portales.mineduc.cl/usuarios/convivencia_escolar/doc/201103021416340.Politica_de_Participacion_de_Padres_ Madres_y_Apoderados_en_el_Sistema_Escolar.pdf

Mineduc. (2011a). Convivencia Escolar. Extraído el 22 de julio de 2013 desde http:// www.convivenciaescolar.cl/index2.php?id_portal=50\&id_seccion=4010\&id_contenido $=1$

Mineduc. (2011b). Conviviendo Mejor en la Escuela y el Liceo: Orientaciones para abordar la Convivencia Escolar en las Comunidades Educativas. Recuperado de: http://portales.mineduc.cl/usuarios/convivencia_escolar/doc/201203291223210. Conviviendo.pdf

Mineduc. (2013) Gestión de la Buena Convivencia. Orientaciones para el encargado de Convivencia Escolar y equipos de Liderazgo Educativo. Recuperado de: http:// portales.mineduc.cl/usuarios/convivencia_escolar/doc/201309091630460.GestiondelaBuenaConvivencia.pdf

Mora, M. E., \& Estepa, J. (2012). La democracia como fundamento para la enseñanza de la participación. En N. De Alba, F. García Pérez, \& A. Santisteban, Educar para la participación ciudadana en la enseñanza de las Ciencias Sociales (pp. 93-100). Sevilla, España: Asociación Universitaria de Profesorado de Ciencias Sociales.

Núñez, I. (2004). La identidad de los docentes. Una mirada histórica en Chile. Santiago: Ponencia presentada al XIV Congreso de la Asociación Mundial de Ciencias de la Educación. 
Obando, G (2008). La participación docente en la toma de decisiones desde la visión de la micropolítica. Educación, 17(32), 87-108.

Oraisón, M., \& Pérez, A. M. (2006). Escuela y participación: el difícil camino de la construcción de ciudadanía. Revista Iberoamericana de Educación, 42, 15-29.

Ortega, R. (2012). Convivencia escolar: Dimensiones y abordaje. Documento de trabajo del Núcleo de Educación del Departamento de Sociología de la Universidad de Chile.

Palma, R. (2011). Estudio comparativo sobre experiencias educativas de convivencia escolar y prevención de la violencia escolar en américa latina y el caribe.

Pérez, A. I. (2009). Menudos ciudadan@s. Madrid, España: Pearson Educación.

Perrenoud, P. (1996). La construcción del éxito y del fracaso escolar: Hacia un análisis del éxito, del fracaso y de las desigualdades como realidades construidas por el sistema escolar. 2a ed. Madrid, España: Morata.

Prieto, M. (2001). La investigación en el aula: una tarea posible. Valparaíso, Chile: Ediciones Universitarias, Universidad Católica de Valparaíso.

Quintana, A. (2006). Metodología de investigación científica cualitativa. En Quintana, A. y Montgomery, W. (Eds.), Psicología: Tópicos de actualidad. Lima: UNMSM.

Ramírez, T. (2007). Estudio de la oferta laboral docente. Tesis de postgrado. Pontificia Universidad Católica de Chile, Santiago, Chile.

Rodas, M. T. (2003). Participación y democracia. Documento Valoras UC. Recuperado de: http://valoras.uc.cl/images/centro-recursos/equipo/ValoresEticaYDesarrolloSocioemocional/Documentos/Participacion-y-democracia.pdf

Ruz, J. (2006) Convivencia y Calidad de la Educación. Organización de Estados Iberoamericanos para la Ciencia y la Educación (OEI). Pg. 2247 - 262

Santos Guerra, M. A. (2004). Arte y parte: Desarrollar la democracia en la escuela. Argentina: Editorial Homo Sapiens.

Tuvilla, J. (2007). Convivencia escolar y resolución pacífica de los conflictos. Consejería de Educación y Ciencia. Junta de Andalucía.

Unesco. (1995). Declaración de la 44ª reunión de la Conferencia Internacional de Educación. Disponible en http://www.unesco.org/cpp/sp/declaraciones/educacion. htm

Unicef. (2011). Violencia Escolar en América Latina y el Caribe. Superficie y Fondo. Recuperado de: https://www.unicef.org/lac/violencia_escolar_OK.pdf

Valencia Murcia, F., \& Mazuera, V. (2006). La figura del manual de convivencia en la vida escolar. Elementos para su comprensión. Revista Científica Guillermo de Ockham, 4(1), 119-131.

Vicente de Abad, J. (2010). 7 Ideas claves: Escuelas sostenibles en Convivencia. Barcelona: Editorial Grao.

Yoneyama, S., \& Naito, A. (2003). Problems with the paradigm: The school as a factor in understanding bullying (With special reference to Japan). British Journal of Sociology of Education, 24(3), 315-330. 